\title{
Harmonic Signal Analysis for Magnetic Nanoparticle Imaging
}

\author{
N. B. OTHMAN, T. TSUBAKI, D. KITAHARA, T. YOSHIDA, and K. ENPUKU
}

Department of Electrical and Electronic Engineering, Kyushu University, 744 Motooka, Nishi-ku, Fukuoka 819-0395, Japan

\begin{abstract}
A harmonic signal analysis of magnetic nanoparticles (MNPs) used as tracers in magnetic particle imaging (MPI) was conducted. The harmonic spectrum from three types of immobilized MNPs namely, Resovist, fluidMAG, and SupraBead, were measured at excitation fields of $1.4 \mathrm{mT}$ and $28 \mathrm{mT}$ with a frequency of $10 \mathrm{kHz}$. Resovist showed the richest spectrum, followed by fluidMAG and SupraBead. These different harmonic properties among samples were analyzed on the basis of the magnetic moment $m$ and anisotropy energy $E_{\mathrm{B}}$ of the MNPs. We show the conditions of $m$ and $E_{\mathrm{B}}$ required for harmonic signal generation. As $m$ and $E_{\mathrm{B}}$ are widely distributed within MNPs, a fraction of MNPs satisfies the $m$ and $E_{\mathrm{B}}$ conditions and contributes to the harmonic signals. Resovist shows the largest such fraction satisfying these conditions. This is reflected in the experimentally obtained rich harmonic spectrum of Resovist. The experimental data was found to agree well with the numerical simulation results based on the Landau-Lifshitz-Gilbert equation when the distributions of $m$ and $E_{\mathrm{B}}$ within the MNPs were taken into account. These findings may serve as an important basis in the optimization of MNPs for MPI.
\end{abstract}

Key Words: Magnetic nanoparticles, Harmonic signal, Nonlinear magnetization, Magnetic moment, Relaxation time

\section{INTRODUCTION}

Magnetic particle imaging (MPI) is a new technique for the quick 3D imaging of the distributions of polymer-coated magnetic nanoparticles (MNPs), especially in in-vivo diagnostic procedures such as those for detecting cancer cells in the human body [1]. Previous high-sensitivity imaging systems have been based on various detection methods employing AC susceptibility, magnetic relaxation, and harmonic signal detection from the nonlinearity of the magnetization $(M-H)$ curve [1]-[6].

As MPI generally utilizes harmonic signals, it is important to select MNPs that can generate strong harmonic signals. However, MNPs show a wide variety of magnetic properties, depending on their magnetic moment $m$ and anisotropy energy $E_{\mathrm{B}}$ [7]. Therefore, it is important to establish a clear quantitative relationship between these parameters and harmonic signals in order to determine suitable MNPs for MPI applications.

In this study, we investigate the properties of the harmonic signals generated by MNPs. First, we measure the harmonic signals from three types of commercial MNPs, namely, Resovist ${ }^{\circledR}$, fluidMAG, and SupraBead. Then, samples with different harmonic properties are analyzed using the $m$ and $E_{\mathrm{B}}$ of MNPs. We also perform a numerical simulation for harmonic spectrum based on the Landau-Lifshitz-Gilbert $(L L G)$ equation. A good agreement is obtained between the measured spectrum and the numerical simulation.

\section{EXPERIMENTAL}

In this work, we investigated the properties of the harmonic signals generated by three types of commercial MNPs, namely, Resovist ${ }^{\circledR}$ (FUJIFILM RI Pharma), fluidMAG (Chemicell), and SupraBead (Recenttec) MNPs. The mean hydrodynamic diameters $D_{\mathrm{H}}$ for these MNPs are approximately 60, 50, and $100 \mathrm{~nm}$, respectively. We prepared an immobilized MNP sample by diluting the MNPs with glycerol.

First, we measured the harmonic signals of immobilized MNPs using a measurement system developed in our laboratory. In the experiment, we applied a small excitation field $B_{1}=1.4 \mathrm{mT}$ and a large excitation field $B_{2}=28 \mathrm{mT}$ with the frequency of $f=10 \mathrm{kHz}$ to the samples. A number of $k^{\text {th }}$ harmonic signals were extracted using a lock-in-amplifier.

In addition, we measured the $M-H$ curve of MNPs in the suspension, with the aim of obtaining the distribution of $m$ within MNPs. The frequency dependence of the AC susceptibility was also measured under a measurement frequency of $f$ $=10 \mathrm{~Hz}-1 \mathrm{MHz}$ to obtain the distribution of the $E_{\mathrm{B}}$. Details of the estimation of $m$ and $E_{\mathrm{B}}$ are described elsewhere [8]. 


\section{RESULTS AND DISCUSSION}

\section{A. Harmonic Signal Measurement}

Figure 1(a) shows the harmonic spectrum measured for the three MNPs. The open and closed symbols show the results for the excitation fields of $B_{1}=1.4 \mathrm{mT}$ and $B_{2}=28 \mathrm{mT}$, respectively. As shown, rich harmonic spectra were obtained for the case of strong excitation fields, while the harmonic signal rapidly decreased as the strength of the field decreased.

Figure 1(b) shows the normalized harmonic signal for each MNP. As shown, Resovist had the richest spectrum, followed by the fluidMAG and SupraBead MNPs. Further, the decay of the harmonic spectra was the weakest for the case of Resovist.

\section{B. Harmonic Signal Analysis}

It is well known that the nonlinear magnetization properties of MNPs can be expressed by the Langevin function [9].

$$
\langle m\rangle /\left(M_{s} V_{c}\right)=\operatorname{coth}(\xi) 1 / \xi
$$

where $<m>$ is the mean magnetic moment of MNPs in the direction of applied field $H, \quad \xi=\mu_{0} m H / k_{B} T$ is the normalized field, $M_{\mathrm{s}}$ is the saturation magnetization, $V_{\mathrm{c}}$ is the magnetic core volume, $k_{\mathrm{B}}$ is the Boltzmann constant, and $T$ is the absolute temperature. Harmonic signals are found to appear for $\xi>1$, owing to the nonlinearity of the Langevin function. Accordingly, harmonic signals can be obtained for MNPs satisfying the condition of $\xi>1$.

The response time of MNPs, $\tau_{\text {eff }}$, can be given by [10]

$$
\left.\tau_{\text {eff }}=2 \tau_{N 0} \exp \sigma(1+h)^{2}+\sigma(1-h)^{2}\right] /\left\{\exp \left[\sigma(1+h)^{2}\right]+\exp \left[\sigma(1-h)^{2}\right]\right\}
$$

where $\tau_{\mathrm{N} 0}=10^{-9} \mathrm{~s}, \sigma=E_{\mathrm{B}} / k_{\mathrm{B}} T, h=\mu_{0} H M_{\mathrm{s}} / 2 K=H / H_{\mathrm{c}}$ is the normalized field, $H_{\mathrm{c}}=2 K / \mu_{0} M_{\mathrm{s}}$ is the coercive field, and $K=$ $E_{\mathrm{B}} / V_{\mathrm{c}}$ is the anisotropy energy constant. It should be noted that Eq. (2) gives the effective Neel relaxation time when the effect of applied field is taken into account. When the applied field is small, Eq. (2) becomes the Neel relaxation time as $\tau_{\text {eff }}=\tau_{N}=\tau_{N 0} \exp \left(E_{B} / k_{B} T\right)$ [11].

MNPs are found to respond to the excitation field when $\omega \tau_{\text {eff }}<1$, where $\omega$ is the angular frequency of the excitation field. Conversely, at high frequencies of $\omega \tau_{\text {eff }}>1$, MNPs cannot respond to the excitation field. Hence, harmonic signals can be obtained for MNPs satisfying the condition of $\omega \tau_{\text {eff }}<1$.

Therefore, we report that harmonic signals can be obtained when both conditions of $\xi>1$ and $\omega \tau_{\text {eff }}<1$ are satisfied. Since these conditions are determined by the value of $m$ and $E_{\mathrm{B}}$ of MNPs, it is necessary to obtain the relationship between them in order to estimate the fraction of MNPs that contributes to harmonic signals. We have shown that their relationship can be obtained by analyzing the $M-H$ curve and the frequency dependence of the AC susceptibility of MNPs [8]. In Fig. 2(a), the obtained relationship between $m$ and $\sigma=E_{\mathrm{B}} / k_{\mathrm{B}} T$ is shown for Resovist MNPs. As shown, $\sigma$ increases almost linearly with $m$. We could evaluate $K=3 \mathrm{~kJ} / \mathrm{m}^{3}$ and $\mu_{0} H_{\mathrm{c}}=16.8 \mathrm{mT}$, where $M_{\mathrm{s}}=364 \mathrm{kA} / \mathrm{m}$ was obtained from the $M-H$ curve.

We now discuss the fraction of MNPs that contributes to harmonic signals. The double arrow symbols in Fig. 2(a) show the fraction of Resovist MNPs that satisfies the conditions of $\xi>1$ and $\omega \tau_{\text {eff }}<1$. This fraction contributes to harmonic signals. As shown, this fraction is small for the weak applied field of $B_{1}$, whereas it is large for the strong field of $B_{2}$.

In Fig. 2(b), distributions of $m$ within MNPs are shown for three types of MNPs; this distribution was obtained by analyzing the $M-H$ curve [8]. Vertical axis in Fig. 2(b) represents the value of $n m^{2}$, where $n(m)$ is the number density of MNPs having a moment $m$. As shown, the value of $m$ is widely distributed within MNPs. The double arrow symbols indicate the fraction of MNPs that satisfies the conditions of $\xi>1$ and $\omega \tau_{\text {eff }}<1$ when the $B_{2}$ field is applied. As shown, Resovist has a larger fraction as compared to fluidMAG and SupraBead. This justifies the rich spectrum that is obtained experimentally of Resovist, as compared to the others. 


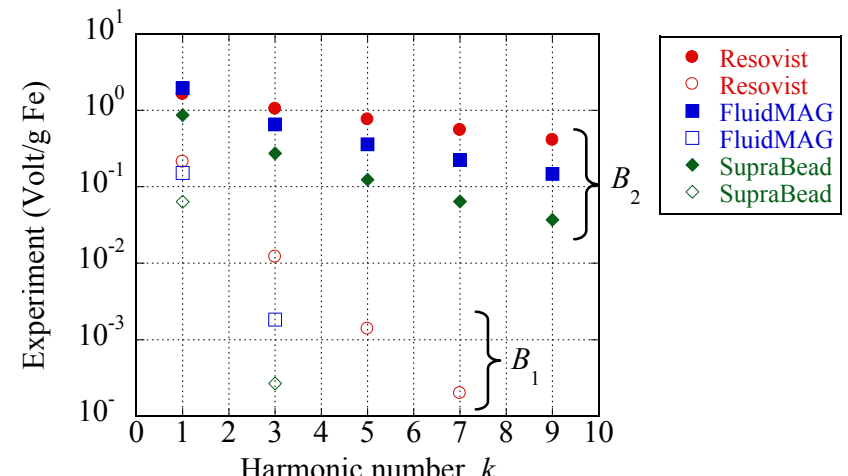

(a)

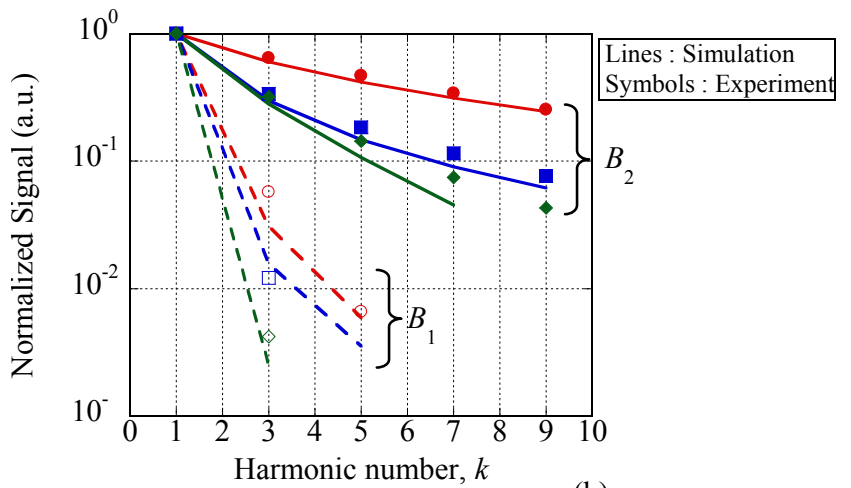

(b)

Fig. 1 (a) Harmonic spectra of Resovist, fluidMag, and SupraBead MNPs under $B_{1}$ and $B_{2}$ excitation, and (b) Comparison of normalized signals between measurement and numerical simulation results.
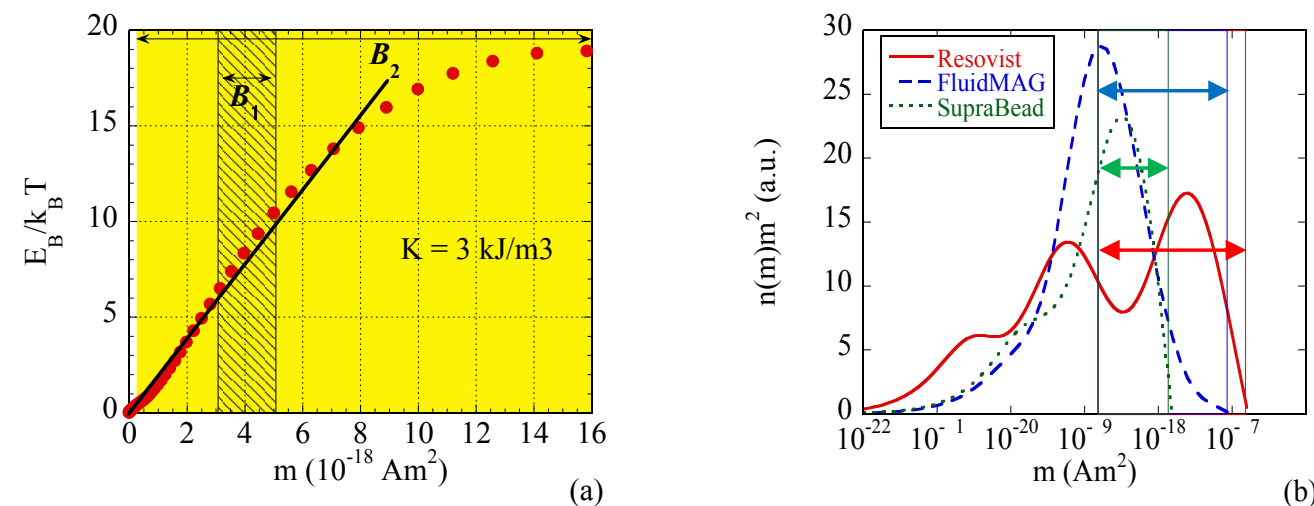

Fig. 2 (a) $m$ - $\sigma$ relationship of Resovist, and (b) Distribution of magnetic moment $m$ within MNPs. Double arrow symbols indicate the fraction of MNPs that contributes to the harmonic signals at $B_{2}=28 \mathrm{mT}$.

\section{Numerical Simulation}

Finally, we performed the numerical simulation for harmonic spectra based on the $L L G$ equation given by [12]

$$
d \vec{m} / d t=\gamma \vec{m} \times\left[\vec{B}_{e f}+\vec{B}_{T H}\right\rfloor-(\gamma \lambda / m) \vec{m} \times\left\{\vec{m} \times\left[\vec{B}_{e f f}+\vec{B}_{T H}\right]\right\}
$$

where $\vec{m}$ is the magnetic moment, $\gamma$ is the gyromagnetic ratio, and $\lambda$ is a dimensionless damping coefficient. $\vec{B}_{e f f}=\vec{B}_{a p p}+\vec{B}_{a}$ is the effective field that combines the effects of the magnetic-anisotropy field $\vec{B}_{a}=\left(2 K V_{c} / m\right)[(\vec{m} \cdot \vec{n}) / m] \vec{n}$ and the applied field $\vec{B}_{a p p}$. $\vec{n}$ is a unit vector along the symmetry axis, and $\vec{B}_{T H}$ is the fluctuating field, which causes the fluctuations of the magnetic moment orientation due to thermal noise.
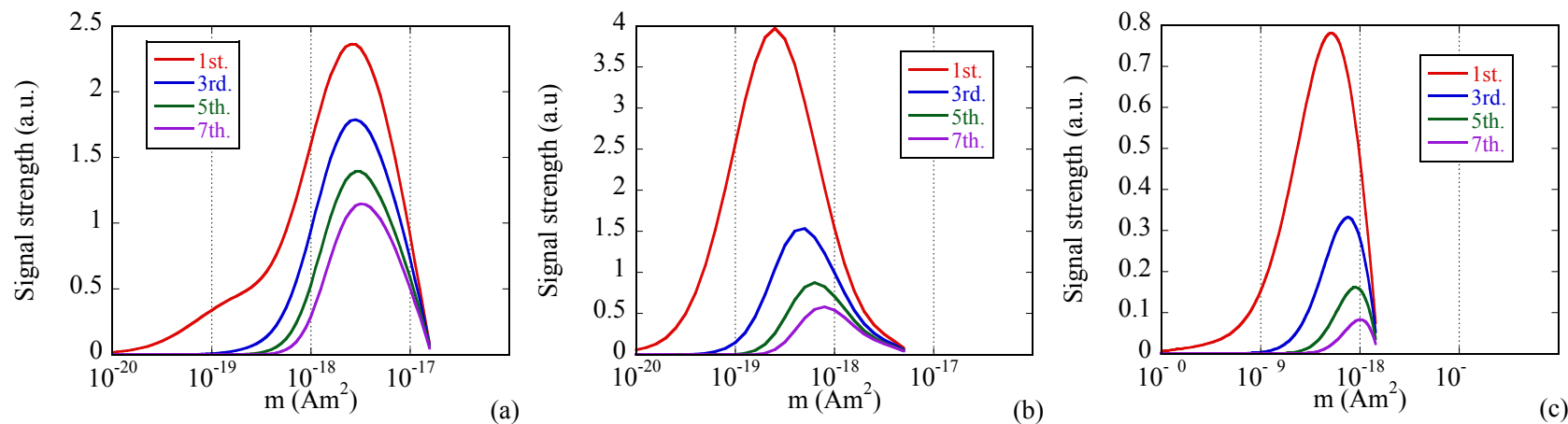

Fig. 3 Harmonic signal strength generated by each $m$ distributed in (a) Resovist, (b) fluidMAG, and (c) SupraBead MNPs. Harmonic signals with $k=1$ to 7 are shown. 
Considering the $m$ and $E_{\mathrm{B}}$ distribution within MNPs, we used the $m$ - $\sigma$ relationship in Fig. 2(a) to calculate the magnetization dynamic of the MNPs. The spectra obtained from simulation are indicated as solid and dashed lines in Fig. 1(b). As shown, the experimental data agreed well with the simulation results.

Using the numerical simulation, we can quantitatively estimate what portion of $m$, which distributed in MNPs as shown in Fig. 2(b), is responsible for the generation of harmonic signals. In Fig. 3, calculated values of the harmonic signals generated by each $m$ are shown when $B_{2}=28 \mathrm{mT}$ is applied. As shown in Fig. 3(a), in the case of Resovist, all MNPs with $3.4 \times 10^{-19}<m<1.58 \times 10^{-17}$ generated the harmonic signals as well as the fundamental signal. In the case of fluidMAG, on the other hand, fraction of $m$ that generated the harmonic signal is much smaller than that for the fundamental signal, as shown in Fig. 3(b). Moreover, the fraction of $m$ decreased rapidly with the increase of the harmonic number. This explains the quick decay of its harmonic spectra as shown in Fig. 1(a). Similar result was obtained in the case of SupraBead, as shown in Fig. 3(c).

\section{CONCLUSIONS}

In summary, we studied the harmonic signals of MNPs to find the optimal MNPs for the MPI system. From the harmonic spectrum measurement, Resovist showed the richest spectrum, followed by fluidMAG and SupraBead. We showed that the MNPs satisfying the conditions of $\xi>1$ and $\omega \tau_{\text {eff }}<1$ contribute to harmonic signals. The difference among the harmonic spectra from the three MNPs could be explained by the difference in the MNP fractions satisfying these conditions. Finally, we found a good agreement between the experimental data and the $L L G$-based-numerical simulation. The portion of $\mathrm{m}$ which is responsible for the harmonic signals generation in each MNPs could be studied in details using the numerical simulation results.

\section{REFERENCES}

[1] E. U. Saritas, P. W. Goodwill, L. R. Croft, J. J. Konkle, K. Lu, B. Zheng, et. al., J. Magn. Reson. 229, 116 (2013).

[2] B. Gleich and J. Weizenecker, Nature, 435, 1214 (2005).

[3] S. Tanaka, H. Ota, Y. Kondo, Y. Tamaki, S. Kobayashi and S. Noguchi, IEEE Trans. Appl. Supercond. 13, 377 (2003).

[4] H. J. Hathaway, K. S. Butler, N. L. Adolphi, D. M. Lovato, R. Belfon, D. Fegan, et al., Breast Cancer Research, 13, R108 (2011).

[5] P. W. Goodwill, G. C. Scott, P. P. Stang, and S. M. Conolly, IEEE Trans. Med. Imag. 28, 1231 (2009).

[6] N. B. Othman, T. Tsubaki, T. Yoshida, K. Enpuku, and A. Kandori, IEEE Trans. Magn. 48, 3776 (2012).

[7] T. Yoshida, N. B. Othman, T. Tsubaki, J. Takamiya, and K. Enpuku, IEEE Trans. Magn. 48, 3788 (2012).

[8] T. Yoshida, K. Enpuku, F. Ludwig, J. Dieckhoff, T. Wawrzik, A. Lak, et al., Springer Proc. in Phys. 140, 3 (2012).

[9] S. Chikazumi et al., "Physics of Magnetism", Wiley, New York, U.S.A, 1964.

[10] K. Enpuku, K. Inoue, and K. Soejima, Jpn. J. Appl. Phys. 44, 149 (2005).

[11] L. Néel, $A d v$. in Phys. 4, 191 (1995).

[12] J. Luis, G. Palacois, and F. J. Lazaro, Physical Rev. B, 58, 14937 (1998). 